\title{
On displacement based non-local models for non-linear vibrations of thin nano plates
}

\author{
Tomás R. C. Chuaqui ${ }^{1}$, Pedro Ribeiro ${ }^{1, *}$ \\ ${ }^{1}$ DEMec/INEGI, Faculdade de Engenharia da Universidade do Porto, Portugal
}

\begin{abstract}
This paper addresses the formulation of displacement based, non-linear, plate models adopting Eringen's non-local elasticity, to study the modes of vibration of thin, nano plates. Plate models governed by ordinary differential equations of motion with generalized displacements as unknowns have some advantages over mixed type formulations, but difficulties arise in the development of such non-linear models when non-local effects are taken into account. To circumvent those difficulties, approximations of debatable justification can be imposed. Different approximations are discussed here and the accuracy of the best non-local, non-linear displacement based model achieved is put to test, by carrying out comparisons with a model based on Airy's stress function.
\end{abstract}

\section{Introduction}

Micro and nano systems are rapidly evolving and increasingly attracting the interest of researchers and engineers. Vibrating plates frequently appear as components in these systems. In particular, due to their promising properties and possible applications, substantial attention has been given to graphene sheets $[1,2]$.

Atomistic simulations have been extensively used in nano mechanics [3], but they require high computational resources [4]. Continuum modelling techniques do not suffer from this handicap and classical elasticity theories have been applied in attempts to replicate the mechanical behaviour of components used in nanotechnology. However, some analyses indicate that classical elasticity theories are not always able to correctly predict the behaviour of nanostructures, because they miss small scale effects $[5,6]$. In order to solve this problem, nonlocal continuum theories that take into account the interatomic forces and the internal length scales have been applied. In particular, Eringen's non-local elasticity theory $[7,8]$ has been used as the basis of many studies [5, 9-13].

Nano plates can achieve large displacements, in comparison to their dimensions, inducing geometric nonlinearities, which lead to an effective stiffening of the plate, consequently altering the natural frequencies and mode shapes of vibration. In the geometrical non-linear regime, the non-local differential constitutive equations cause difficulties in plate models, because of the coupling between the diverse stress resultants [9]. A way to circumvent this issue and arrive at displacement based workable models, is to neglect some non-linear non-local terms of the equilibrium equations, but the rationale for neglecting those terms and the ensuing errors are issues of concern. Using Airy's stress function - hence not following a totally displacement based approach - offers another solution to sidestep the aforementioned coupling [12-15].

In this paper, the implementation of displacement based non-linear and non-local models of nano plates is discussed. The models are based on Kirchhoff's thin plate theory and include Von Kármán's geometrical nonlinear terms [16]. To investigate their performance, the backbone curves of a nano plate computed using them are compared with the curves obtained using a model based on Airy's stress function [15].

\section{Displacement based models}

\subsection{Non-local equations of motion}

\subsubsection{Equilibrium equations}

The displacement components in directions $x, y$ and $z$ represented by $u, v$ and $w$, respectively - are given by

$$
u=u^{0}-z \frac{\partial w^{0}}{\partial x}, v=v^{0}-z \frac{\partial w^{0}}{\partial x}, w=w^{0}
$$

where $u^{0}, v^{0}$ and $w^{0}$ represent the displacement components of points on the middle plane (plane Oxy). The strain-displacement relations are

$$
\left\{\begin{array}{c}
\varepsilon_{x} \\
\varepsilon_{y} \\
\gamma_{x y}
\end{array}\right\}=\left\{\begin{array}{c}
\frac{\partial u^{0}}{\partial x} \\
\frac{\partial v^{0}}{\partial y} \\
\frac{\partial u^{0}}{\partial y}+\frac{\partial v^{0}}{\partial x}
\end{array}\right\}+\left\{\begin{array}{c}
\frac{1}{2}\left(\frac{\partial w^{0}}{\partial x}\right)^{2} \\
\frac{1}{2}\left(\frac{\partial w^{0}}{\partial y}\right)^{2} \\
\frac{\partial w^{0}}{\partial x} \frac{\partial w^{0}}{\partial y}
\end{array}\right\}-z\left\{\begin{array}{c}
\frac{\partial^{2} w^{0}}{\partial x^{2}} \\
\frac{\partial^{2} w^{0}}{\partial y^{2}} \\
2 \frac{\partial^{2} w^{0}}{\partial x \partial y}
\end{array}\right\}
$$

\footnotetext{
* Corresponding author: pmleal@,fe.up.pt
} 
with the remaining strains equal to zero.

The following equations of motion correspond to the free vibration case and can be derived, for example, applying Hamilton's principle

$$
\begin{gathered}
\frac{\partial N_{x x}}{\partial x}+\frac{\partial N_{x y}}{\partial y}=\rho h \frac{\partial^{2} u^{0}}{\partial t^{2}} \\
\frac{\partial N_{x y}}{\partial x}+\frac{\partial N_{y y}}{\partial y}=\rho h \frac{\partial^{2} v^{0}}{\partial t^{2}} \\
\frac{\partial^{2} M_{x x}}{\partial x^{2}}+2 \frac{\partial^{2} M_{x y}}{\partial y \partial x}+\frac{\partial^{2} M_{y y}}{\partial y^{2}}+N_{\text {nlinear }}= \\
\rho h \frac{\partial^{2} w^{0}}{\partial t^{2}}-\rho h^{3}\left(\frac{\partial^{4} w^{0}}{\partial t^{2} \partial x^{2}}+\frac{\partial^{4} w^{0}}{\partial t^{2} \partial y^{2}}\right)
\end{gathered}
$$

where

$$
\begin{aligned}
& N_{\text {nlinear }}=\frac{\partial}{\partial x}\left(N_{x x} \frac{\partial w^{0}}{\partial x}+N_{x y} \frac{\partial w^{0}}{\partial y}\right)+ \\
& +\frac{\partial}{\partial y}\left(N_{x y} \frac{\partial w^{0}}{\partial x}+N_{y y} \frac{\partial w^{0}}{\partial y}\right)
\end{aligned}
$$

The effect of rotary inertia will be neglected. In-plane inertia can also be neglected, because its importance in periodic oscillations of thin plates is, in general, small [17]. Due to the latter simplification, equation (6) leads to

$$
N_{\text {nlinear }}=N_{x x} \frac{\partial^{2} w^{0}}{\partial x^{2}}+2 N_{x y} \frac{\partial^{2} w^{0}}{\partial y \partial x}+N_{y y} \frac{\partial^{2} w^{0}}{\partial y^{2}}
$$

\subsubsection{Non-local elasticity}

So far, non-local effects were not introduced. According to $[8,18]$, the equations of motion for a generic nonlocal linear elastic solid, are:

$$
t_{i j, j}+f_{i}=\rho \ddot{u}_{i}
$$

with $\rho, f_{i}, \ddot{u}_{i}$ designating the mass density, applied forces and the acceleration vector respectively. The stress tensor $t_{i j}$ in Eringen's non-local elasticity is defined by the following volume integral over the region $V$ occupied by the body

$$
t_{i j}(\mathbf{x})=\int_{V} \alpha\left(\left|\mathbf{x}^{\prime}-\mathbf{x}\right|\right) \sigma_{i j}\left(\mathbf{x}^{\prime}\right) \mathrm{d} v\left(\mathbf{x}^{\prime}\right)
$$

where $\mathbf{x}$ is a reference point in the body, $\alpha\left(\left|\mathbf{x}^{\prime}-\mathbf{x}\right|\right)$ is the non-local kernel function [8] and $\sigma_{i j}\left(\mathbf{x}^{\prime}\right)$ is the local, or stress tensor of the classical theory of elasticity at any point $\mathbf{x}^{\prime}$. Based on this theory, the stress at an arbitrary point is not only a function of the components of strain at that point but also a function of strain at all other points of the structure. It is a size-dependent theory; as the dimensions increase - and, consequently, the internal characteristic length becomes much smaller than the external or macroscopic length of the structure - it approaches the traditional, local, theory of elasticity.
Instead of writing equation (8) as an integro-partial differential equation using (9), it is possible to write it in partial differential form making [8]

$$
\left[1-\left(e_{0} a_{i}\right)^{2} \nabla^{2}\right] t_{i j}=\sigma_{i j}
$$

where $\nabla^{2}$ is the Laplacian, $a_{i}$ is an internal characteristic length (lattice parameter, granular size or molecular diameter), and $e_{0}$ is an empirical material constant. Employing the local constitutive equations, (10) leads to

$$
\left[1-\left(e_{0} a_{i}\right)^{2} \nabla^{2}\right] t_{i j}=C_{i j k l} \varepsilon_{k l}
$$

where $\mathrm{C}_{i j k l}$ are the components of the fourth order stiffness or elasticity tensor, and $\varepsilon_{k l}$ is the strain tensor. In the rest of this section, a non-local parameter $\mu=\left(e_{0}\right.$ $\left.a_{i}\right)^{2}$ is employed, for the sake of simplicity.

Now, we extend the non-local elasticity theory to the Kirchhoff plate, and write the constitutive equations in terms of stress resultants as [9]

$$
\begin{aligned}
& \left\{\begin{array}{l}
N_{x x} \\
N_{y y} \\
N_{x y}
\end{array}\right\}-\mu \nabla^{2}\left\{\begin{array}{l}
N_{x x} \\
N_{y y} \\
N_{x y}
\end{array}\right\}= \\
& =\left[\begin{array}{ccc}
A_{11} & A_{12} & 0 \\
A_{12} & A_{22} & 0 \\
0 & 0 & A_{66}
\end{array}\right]\left\{\begin{array}{c}
\frac{\partial u^{0}}{\partial x}+\frac{1}{2}\left(\frac{\partial w^{0}}{\partial x}\right)^{2} \\
\frac{\partial v^{0}}{\partial y}+\frac{1}{2}\left(\frac{\partial w^{0}}{\partial y}\right)^{2} \\
\frac{\partial u^{0}}{\partial y}+\frac{\partial v^{0}}{\partial x}+\frac{\partial w^{0}}{\partial x} \frac{\partial w^{0}}{\partial y}
\end{array}\right\}, \\
& \left\{\begin{array}{l}
M_{x x} \\
M_{y y} \\
M_{x y}
\end{array}\right\}-\mu \nabla^{2}\left\{\begin{array}{l}
M_{x x} \\
M_{y y} \\
M_{x y}
\end{array}\right\}= \\
& =-\left[\begin{array}{ccc}
D_{11} & D_{12} & 0 \\
D_{12} & D_{22} & 0 \\
0 & 0 & D_{66}
\end{array}\right]\left\{\begin{array}{c}
\frac{\partial^{2} w^{0}}{\partial x^{2}} \\
\frac{\partial^{2} w^{0}}{\partial y^{2}} \\
2 \frac{\partial^{2} w^{0}}{\partial x \partial y}
\end{array}\right\},
\end{aligned}
$$

where coefficients $A_{i j}$ and $D_{i j}$ are

$$
A_{i j}=h Q_{i j}, \quad D_{i j}=\frac{h^{3}}{12} Q_{i j}
$$

with the plane-stress reduced stiffnesses $Q_{i j}$ as given, for example, in [19].

Applying the differential operator $\mathcal{L}=1-\mu \nabla^{2}$ to equations (3)-(5), and the constitutive relations (12) and (13), we arrive at the equations of motion for non-local Kirchhoff plates with Von Kármán's type non-linearity

$$
\begin{aligned}
& \left(1-\mu \nabla^{2}\right)\left(\frac{\partial^{2} M_{x x}}{\partial x^{2}}+2 \frac{\partial^{2} M_{x y}}{\partial y \partial x}+\frac{\partial^{2} M_{y y}}{\partial y^{2}}\right)+ \\
& +\left(1-\mu \nabla^{2}\right) N_{\text {nlinear }}=\left(1-\mu \nabla^{2}\right)\left(\rho h \frac{\partial^{2} w^{0}}{\partial t^{2}}\right)
\end{aligned}
$$




\subsection{Simplifications of displacement based non- linear, non-local equations of motion}

The difficulty in displacement based non-linear, nonlocal plate models resides in dealing with term

$$
\begin{aligned}
& \left(1-\mu \nabla^{2}\right) N_{\text {nlinear }}= \\
& \left(1-\mu \nabla^{2}\right)\left(N_{x x} \frac{\partial^{2} w^{0}}{\partial x^{2}}+2 N_{x y} \frac{\partial^{2} w^{0}}{\partial y \partial x}+N_{y y} \frac{\partial^{2} w^{0}}{\partial y^{2}}\right)
\end{aligned}
$$

due to the couplings introduced by the stress resultants. This issue was already mentioned in [9], where the static case is addressed. The following sections provide a detailed description of simplification options available in order to achieve displacement based formulations.

\subsubsection{Neglecting all non-linear terms}

An oversimplifying way to deal with the issue, stated here for the sake of completeness, is to linearise equation (15), which would become

$$
\begin{aligned}
& \left(1-\mu \nabla^{2}\right)\left(\frac{\partial^{2} M_{x x}}{\partial x^{2}}+2 \frac{\partial^{2} M_{x y}}{\partial y \partial x}+\frac{\partial^{2} M_{y y}}{\partial y^{2}}\right)= \\
& \left(1-\mu \nabla^{2}\right)\left(\rho h \frac{\partial^{2} w^{0}}{\partial t^{2}}\right)
\end{aligned}
$$

Now, using equation (13), ones gets

$$
\begin{aligned}
& -D_{11} \frac{\partial^{4} w^{0}}{\partial x^{4}}-\left(2 D_{12}+4 D_{66}\right) \frac{\partial^{4} w^{0}}{\partial x^{2} \partial y^{2}}-D_{22} \frac{\partial^{4} w^{0}}{\partial y^{4}}= \\
& =\left(1-\mu \nabla^{2}\right)\left(\rho h \frac{\partial^{2} w^{0}}{\partial t^{2}}\right)
\end{aligned}
$$

So, if non-linearity is disregarded in a free vibration problem, then non-local effects only change the inertia forces. This approach has been widely implemented, namely to analyse the importance of non-local effects on the linear modes of vibration [20].

\subsubsection{Neglecting all non-local non-linear terms}

Another oversimplifying option at hand consists in directly neglecting terms that are simultaneously nonlocal and non-linear, so that the partial differential equation of motion reads

$$
\begin{aligned}
& \left(1-\mu \nabla^{2}\right)\left(\frac{\partial^{2} M_{x x}}{\partial x^{2}}+2 \frac{\partial^{2} M_{x y}}{\partial y \partial x}+\frac{\partial^{2} M_{y y}}{\partial y^{2}}\right)+N_{\text {nlinear }}^{\text {local }} \\
& =\left(1-\mu \nabla^{2}\right)\left(\rho h \frac{\partial^{2} w^{0}(x, y, t)}{\partial t^{2}}\right)
\end{aligned}
$$

with $N_{\text {nlinear }}$ as in local problems, i.e., given by Eq. (12) with $\mu=0$. As occurred in the former section, in this approach the non-local effects do not influence the stiffness, they would only be considered in the external excitation - which is absent in this paper - and in the inertia forces.
One might also be tempted to implement the approximation described in the following lines. Let's start by writing equation (16) in the ensuing form

$$
\begin{aligned}
& \left(1-\mu \nabla^{2}\right)\left(N_{x x} \frac{\partial^{2} w^{0}}{\partial x^{2}}+2 N_{x y} \frac{\partial^{2} w^{0}}{\partial y \partial x}+N_{y y} \frac{\partial^{2} w^{0}}{\partial y^{2}}\right)= \\
& \left(\left(1-\mu \nabla^{2}\right) N_{x x}\right) \frac{\partial^{2} w^{0}}{\partial x^{2}}+2\left(\left(1-\mu \nabla^{2}\right) N_{x y}\right) \frac{\partial^{2} w^{0}}{\partial y \partial x}+ \\
& +\left(\left(1-\mu \nabla^{2}\right) N_{y y}\right) \frac{\partial^{2} w^{0}}{\partial y^{2}}+\text { o.t. }
\end{aligned}
$$

where o.t. stands by "other terms", which have in common the facts that all contain non-local parameter $\mu$ and either a third or fourth order derivative of $w$.

Using the non-local constitutive relation (12), we have

$$
\begin{aligned}
& \left(\left(1-\mu \nabla^{2}\right) N_{x x}\right) \frac{\partial^{2} w^{0}}{\partial x^{2}}+2\left(\left(1-\mu \nabla^{2}\right) N_{x y}\right) \frac{\partial^{2} w^{0}}{\partial y \partial x}+ \\
& +\left(\left(1-\mu \nabla^{2}\right) N_{y y}\right) \frac{\partial^{2} w^{0}}{\partial y^{2}}= \\
& =\left[A_{11}\left(\frac{\partial u^{0}}{\partial x}+\frac{1}{2}\left(\frac{\partial w^{0}}{\partial x}\right)^{2}\right)+A_{12}\left(\frac{\partial v^{0}}{\partial y}+\frac{1}{2}\left(\frac{\partial w^{0}}{\partial y}\right)^{2}\right)\right] \frac{\partial^{2} w^{0}}{\partial x^{2}} \\
& +2 A_{66}\left(\frac{\partial u^{0}}{\partial y}+\frac{\partial v^{0}}{\partial x}+\frac{\partial w^{0}}{\partial x} \frac{\partial w^{0}}{\partial y}\right) \frac{\partial^{2} w^{0}}{\partial x \partial y}+ \\
& {\left[A_{12}\left(\frac{\partial u^{0}}{\partial x}+\frac{1}{2}\left(\frac{\partial w^{0}}{\partial x}\right)^{2}\right)+A_{22}\left(\frac{\partial v^{0}}{\partial y}+\frac{1}{2}\left(\frac{\partial w^{0}}{\partial y}\right)^{2}\right)\right] \frac{\partial^{2} w^{0}}{\partial y^{2}}}
\end{aligned}
$$

Neglecting the "other terms" in (20), which allows replacing the right hand side of that equation by the right side of equation (21), one arrives at equations of motion that would read as

$$
\begin{aligned}
& -D_{11} \frac{\partial^{4} w^{0}}{\partial x^{4}}-\left(2 D_{12}+4 D_{66}\right) \frac{\partial^{4} w^{0}}{\partial x^{2} \partial y^{2}}-D_{22} \frac{\partial^{4} w^{0}}{\partial y^{4}}+ \\
& +\left[A_{11}\left(\frac{\partial u^{0}}{\partial x}+\frac{1}{2}\left(\frac{\partial w^{0}}{\partial x}\right)^{2}\right)+A_{12}\left(\frac{\partial v^{0}}{\partial y}+\frac{1}{2}\left(\frac{\partial w^{0}}{\partial y}\right)^{2}\right)\right] \frac{\partial^{2} w^{0}}{\partial x^{2}} \\
& \quad+2 A_{66}\left(\frac{\partial u^{0}}{\partial y}+\frac{\partial v^{0}}{\partial x}+\frac{\partial w^{0}}{\partial x} \frac{\partial w^{0}}{\partial y}\right) \frac{\partial^{2} w^{0}}{\partial x \partial y}+ \\
& {\left[A_{12}\left(\frac{\partial u^{0}}{\partial x}+\frac{1}{2}\left(\frac{\partial w^{0}}{\partial x}\right)^{2}\right)+A_{22}\left(\frac{\partial v^{0}}{\partial y}+\frac{1}{2}\left(\frac{\partial w^{0}}{\partial y}\right)^{2}\right)\right] \frac{\partial^{2} w^{0}}{\partial y^{2}}=} \\
& =\left(1-\mu \nabla^{2}\right)\left(\rho h \frac{\partial^{2} w^{0}(x, y, t)}{\partial t^{2}}\right)
\end{aligned}
$$

So, neglecting terms o.t. in equation (20) - which is essentially justified by mathematical convenience -, leads to the removal of all non-local terms related with stiffness, that is, it leads again to equation (19).

\subsubsection{Neglecting some non-local non-linear terms}

In order to consider non-local effects on the stiffness, whilst still arriving at a manageable displacement based formulation, the ensuing reasoning will be followed. 
Consider, by way of example, term $\left(1-\mu \nabla^{2}\right)\left(N_{x x} \frac{\partial^{2} w^{0}}{\partial x^{2}}\right)$ of $\left(1-\mu \nabla^{2}\right) N_{\text {nlinear }}$. Employing the first row of equation (12), the following equality can be written

$$
\begin{aligned}
& \left(1-\mu \nabla^{2}\right)\left(N_{x x} \frac{\partial^{2} w^{0}}{\partial x^{2}}\right)=\left(1-\mu \nabla^{2}\right)\left\{\left[A_{11}\left(\frac{\partial u^{0}}{\partial x}+\frac{1}{2}\left(\frac{\partial w^{0}}{\partial x}\right)^{2}\right)+\right.\right. \\
& \left.\left.A_{12}\left(\frac{\partial v^{0}}{\partial y}+\frac{1}{2}\left(\frac{\partial w^{0}}{\partial y}\right)^{2}\right)\right] \frac{\partial^{2} w^{0}}{\partial x^{2}}\right\}+\left(1-\mu \nabla^{2}\right)\left(\left(\mu \nabla^{2} N_{x x}\right) \frac{\partial^{2} w^{0}}{\partial x^{2}}\right)
\end{aligned}
$$

In the term underlined in equation (23), the non-local effects appear in operator $\left(1-\mu \nabla^{2}\right)$ and multiplying by the Laplacian of the stress resultant. We will neglect this term and later analyse the consequences.

Following this line of thought, the equations of motion become

$$
\begin{aligned}
& -D_{11} \frac{\partial^{4} w^{0}}{\partial x^{4}}-\left(2 D_{12}+4 D_{66}\right) \frac{\partial^{4} w^{0}}{\partial x^{2} \partial y^{2}}-D_{22} \frac{\partial^{4} w^{0}}{\partial y^{4}} \\
& +\left(1-\mu \nabla^{2}\right)\left\{\left[A_{11}\left(\frac{\partial u^{0}}{\partial x}+\frac{1}{2}\left(\frac{\partial w^{0}}{\partial x}\right)^{2}\right)+\right.\right. \\
& \left.A_{12}\left(\frac{\partial v^{0}}{\partial y}+\frac{1}{2}\left(\frac{\partial w^{0}}{\partial y}\right)^{2}\right)\right] \frac{\partial^{2} w^{0}}{\partial x^{2}}+ \\
& 2 A_{66}\left(\frac{\partial u^{0}}{\partial y}+\frac{\partial v^{0}}{\partial x}+\frac{\partial w^{0}}{\partial x} \frac{\partial w^{0}}{\partial y}\right) \frac{\partial^{2} w^{0}}{\partial x \partial y}+ \\
& \left.\left[A_{12}\left(\frac{\partial u^{0}}{\partial x}+\frac{1}{2}\left(\frac{\partial w^{0}}{\partial x}\right)^{2}\right)+A_{22}\left(\frac{\partial v^{0}}{\partial y}+\frac{1}{2}\left(\frac{\partial w^{0}}{\partial y}\right)^{2}\right)\right] \frac{\partial^{2} w^{0}}{\partial y^{2}}\right\} \\
& =\left(1-\mu \nabla^{2}\right)\left(\rho h \frac{\partial^{2} w^{0}(x, y, t)}{\partial t^{2}}\right)
\end{aligned}
$$

\section{The ordinary differential equations of motion and their solution}

The partial differential equations of motion (24) were transformed into ordinary differential equations of motion employing a $p$-version finite element type approach; the details do not fit in this paper, but are given in [21]. The resulting ODEs constitute a system of coupled Duffing type equations, which is, for the sake of simplicity, written as

$$
\begin{aligned}
& {\left[\mathbf{M}+\mathbf{M}_{\mu}\right] \ddot{\mathbf{q}}_{w}(t)+\mathbf{K}^{0} \mathbf{q}_{w}(t)+} \\
& +\left[\mathbf{K}_{\mu}^{2}\left(\mathbf{q}_{w}(t)\right)+\mathbf{K}^{2}\left(\mathbf{q}_{w}(t)\right)\right] \mathbf{q}_{w}(t)=\mathbf{0}
\end{aligned}
$$

where $\mathbf{M}$ is used to represent a mass matrix and $\mathbf{K}$ to represent a stiffness matrix. Superscript ${ }^{0}$ indicates a constant stiffness matrix and superscript ${ }^{2}$ a matrix that is a quadratic function of the generalized transverse displacements, $\mathbf{q}_{w}(t)$. Subscript $\mu$ indicates that the matrix is due to non-local effects.

Provided that modal coupling does not exist and discarding the study of bifurcations [22, 23], a one harmonic approach often provides rather accurate backbone curves. In this case, the vector of generalized displacements is written as:

$$
\left.\mathbf{q}_{w}(t)=\mathbf{u} \cos (\omega t)\right) .
$$

The algebraic system that results from substituting (26) in (25) can be solved in a number of ways. We employ the arc-length continuation method presented by $\mathrm{R}$. Lewandowski in [24].

\section{Numerical results}

\subsection{Local non-linear model}

In order to verify the local part of the computational code, tests were carried out on macroscopic plates, making $\mu=0$ [21]. An example of the tests performed is shown in Table 1, which contains the ratio between the first non-linear natural frequency and the linear one, $\omega / \omega$, computed by the present approach, and ratios presented in [25], [26], obtained by other procedures. The plate is "simply supported" with immovable edges, boundaries we designate as "pinned". Furthermore, it is isotropic and rectangular, with the following properties: $E=205800 \mathrm{MPa}, a=20 \mathrm{~mm}, b=10 \mathrm{~mm}, h=0.1 \mathrm{~mm}$, $v=0.3, \rho=7800 \mathrm{~kg} / \mathrm{m}^{3}$. The results of the present model were obtained using 9 symmetric out-of-plane bidimensional shape functions, and either 25 or 49 , both symmetric and anti-symmetric, in-plane shape functions. Ratio $\omega / \omega$ is calculated for different values of $w_{\max } / h$, which designates the ratio between the amplitude of vibration at $x=y=0$ and the thickness of the plate.

Table 1. Ratio $\omega^{\prime} \omega_{\ell}$., isotropic rectangular plate

\begin{tabular}{|c|c|c|c|c|c|}
\hline$\frac{w_{\max }}{h}$ & $\begin{array}{c}\text { Present } \\
\text { model } \\
(9,25)\end{array}$ & $\begin{array}{c}\text { Present } \\
\text { model } \\
(9,49)\end{array}$ & $\begin{array}{c}\text { Harmonic } \\
\text { balance* } \\
{[26],[27]}\end{array}$ & $\begin{array}{c}\text { Elliptic } \\
\text { function } \\
{[26],[27]}\end{array}$ & $\begin{array}{c}\text { Sarma } \\
\text { et al. } \\
{[25]}\end{array}$ \\
\hline 0.2 & 1.026 & 1.026 & 1.0241 & 1.0241 & 1.0254 \\
\hline 0.4 & 1.095 & 1.096 & 1.0933 & 1.0927 & 1.0975 \\
\hline 0.6 & 1.206 & 1.206 & 1.1998 & 1.1975 & 1.2072 \\
\hline 0.8 & 1.349 & 1.349 & 1.3347 & 1.3292 & 1.3447 \\
\hline 1.0 & 1.518 & 1.518 & 1.4903 & 1.4808 & 1.5023 \\
\hline
\end{tabular}

* These results are given in [26] under label "perturbation method"; they are based on [27], where, apparently, the "perturbation method" is the harmonic balance method with one harmonic.

The results presented in Table 1 are very close to each other, suggesting that the local part of the computational code is correct; maybe we ought to point out that other approaches - not shown - give results that diverge more from the ones presented in Table 1 (see [26]), due to differences in hypothesis assumed or solution methods employed. 


\subsection{Non-local non-linear model}

To analyse the consequences of the approximation suggested in section 2.2.3, results from this model were compared with data given by A.Farajpour et al. in [15]. A square nanoplate, assumed to be on $\mathrm{BiTiO}_{3}-\mathrm{CoFe}_{2} \mathrm{O}_{4}$ composites with the following properties was considered: $a=b=60 \mathrm{~nm}, h=4 \mathrm{~nm}, c_{11}=c_{22}=226 \mathrm{GPa}$, $c_{12}=125 \mathrm{GPa}, c_{13}=124 \mathrm{GPa}, c_{33}=216 \mathrm{GPa}, c_{44}=c_{55}=44.2$ $\mathrm{GPa}$ and $c_{66}=50.5 \mathrm{GPa}$, the remaining values of $c_{i j}$ are zero. $a$ and $b$ represent the width and length of the plate, respectively. Details on the application of the values of elastic stifnesses $c_{i j}$ to arrive at the reduced stiffnesses which appear on the constitutive relation between the non-null stresses and strains in a Kirchhoff plate, where plane stress is assumed - are given, for example, in [15, 16]. The plate is considered to be pinned.

We are specifically comparing with [15] because this work stands on the following accurate assumptions:

- a formulation based on Airy's stress function is adopted, allowing one to derive equations of motion of non-local, geometrically non-linear, plates, without neglecting any of the non-linear terms that stem from a Von Kármán's approach;

- the non-local compatibility equation is used (this is not always the case in the literature, some authors employ a local compatibility equation).

Nonetheless, there are upsetting differences between the way boundary conditions are imposed on Airy's stress function based formulations and on displacement based formulations. In the specific case at hand, whilst we impose that the boundaries are immovable in-plane by enforcing precisely that (note that the origin of the coordinate system coincides with the geometric centre of the plate)

$$
u( \pm a / 2, y, t)=0 \quad v(x, \pm b / 2, t)=0 ，
$$

in [15], immovable boundary conditions of the geometric type are written as

$$
\begin{gathered}
x= \pm a / 2 \Rightarrow \int_{-b / 2}^{b / 2} \int_{-a / 2}^{a / 2} \frac{\partial u(x, y, t)}{\partial x} \mathrm{~d} x \mathrm{~d} y=0 \\
y= \pm b / 2 \Rightarrow \int_{-b / 2}^{b / 2} \int_{-a / 2}^{a / 2} \frac{\partial v(x, y, t)}{\partial y} \mathrm{~d} x \mathrm{~d} y=0
\end{gathered}
$$

This averaging procedure is characteristic of formulations based on stress or force functions [11], but equations (28) and (29) are not strictly the same as equations (27).

In Fig. 1 the non-linear natural frequencies computed by the present approach are compared with the ones of [15]. Different values of the non-local parameter, which is represented by its dimensionless value $\chi=e_{0} a_{i} / a$, were considered, from $\chi=0$ for the results on the top, to $\chi=0.3$ for the results on the bottom.

In the local case $(\chi=0)$, the present model was verified (see Table 1). Nevertheless, there are some dissimilarities between the results of the approach proposed in this paper and the results of ref. [15].These specific differences are reasonably small and have as possible reasons the diverse ways adopted to impose boundary conditions, the different methods of solution of the equations of motion and the fact that the values were read from a graph published in [15].

The simplified displacement based approach of section 2.2.3 is able to predict that increasing the nonlocal effects, increases the hardening spring effect. However, as the non-local parameter and the vibration amplitude rises, the simplified displacement based model progressively deviates from the one based on Airy's stress function. Hence, the simplified displacement based formulation presented in 2.2.3 should not be employed in situations where non-local effects are more prevalent and vibration amplitudes larger.

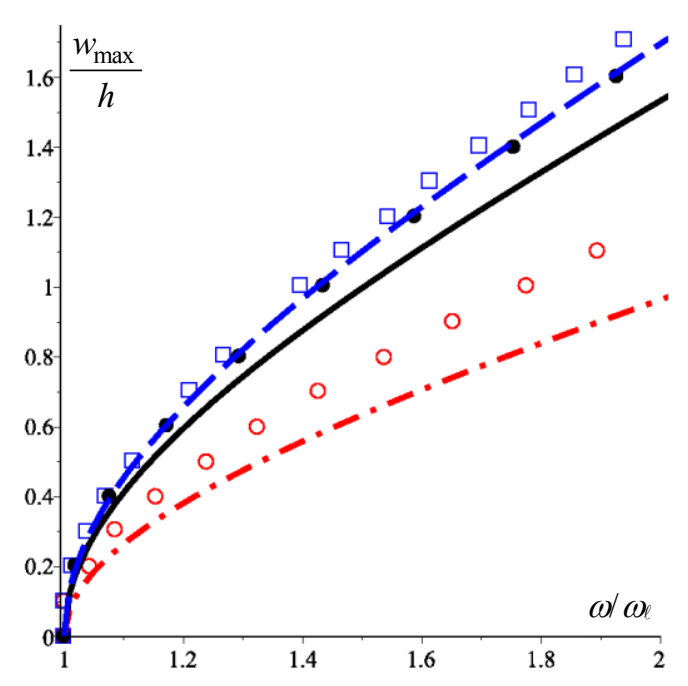

Fig. 1. Backbone curves of the present displacement based approach (lines) and of Airy's stress function based approach [15] (points; read from graph); non-local parameters from topto-bottom (colour online): $\chi=0$ red, $\chi=0.1$ blue, $\chi=0.3$ black.

\section{Final comments}

The implementation of displacement based, non-local formulations, for non-linear vibrations of plates was analysed. The models were based on Eringen's non-local elasticity, Kirchhoff hypothesis and von Kármán type non-linear strain-displacement relations. A displacement based formulation was not achieved without recurring to simplifications. Some of the simplifications that can be employed, and which appear to have been suggested in the literature, lead to a complete removal of non-local terms from the stiffness and were therefore disregarded. An alternative simplification was implemented, where only some non-local terms were neglected.

Natural frequencies of vibration computed by a method based on the latter approach were compared with the frequencies computed via an - apparently more accurate - model where Airy's stress function is applied. The simplified displacement based approach correctly predicted an increasing of the hardening spring effect with the non-local parameter. However, the disagreement between the displacement based model and the one that employs Airy's stress function increases as 
the vibration amplitude and the non-local parameter increase. Therefore, the domain of application of the simplified displacement based procedure is restricted, as it can only be applied if the non-local parameter and the non-linear effects are not large.

The second author gratefully acknowledges the funding of Project NORTE-01-0145-FEDER-000022 - SciTech - Science and Technology for Competitive and Sustainable Industries, cofinanced by Programa Operacional Regional do Norte (NORTE2020), through Fundo Europeu de Desenvolvimento Regional (FEDER).

\section{References}

1. Randviir, E.P., D.A.C. Brownson, and C.E. Banks, Mater. Today 17(9): p. 426-432 (2014).

2. Wang, Q. and B. Arash, Comput. Mater. Sci. 82(Supplement C): p. 350-360 (2014).

3. Eom, K., et al., Phys. Rep. 503: p. 115-163 (2011).

4. Narendar, S., D.R. Mahapatra, and S. Gopalakrishnan, Int. J. Eng. Sci. 49: p. 509522 (2011).

5. Arash, B. and Q. Wang, Comput. Mater. Sci. 51: p. 303-313 (2012).

6. Wang, Q., J. Appl. Phys. 98(12) (2005).

7. Eringen, A.C., Int. J. Eng. Sci. 10: p. 425-435 (1972).

8. Eringen, A., J. Appl. Phys. 54: p. 8 (1983).

9. Reddy, J.N., Int. J. Eng. Sci. 48: p. 1507-1518 (2010).

10. Ansari, R., S. Sahmani, and B. Arash, Phys. Lett. A 375(1): p. 53-62 (2010).

11. Shen, L., H.-S. Shen, and C.-L. Zhang, Comput. Mater. Sci. 48(3): p. 680-685 (2010).

12. Jomehzadeh, E. and A.R. Saidi, Comput. Mater. Sci. 50(3): p. 1043-1051 (2011).

13. Jomehzadeh, E., A.R. Saidi, and N.M. Pugno, Phys. E: Low-dimensional Systems and Nanostructures 44(10): p. 1973-1982 (2012).

14. Li, H.B., et al., J. Sound Vib. 358: p. 285-300 (2015).

15. Farajpour, A., et al., Compos. Struct. 140: p. 323-336 (2016).

16. Chia, C.Y., Nonlinear Analysis of Plates (McGraw-Hill Int. Book Company, 1980).

17. Ribeiro, P., Compos. Sci. Technology 69(2): p. 176-185 (2009).

18. Lu, P., et al., Proc. R. Soc. A 463: p. 32253240 (2007).

19. Reddy, J.N., Mechanics of Laminated Composite Plates and Shells: Theory and Analysis (CRC Press, Boca Raton, 2004).
20. Thai, H.-T., et al.,. Compos. Struct. 177(Supplement C): p. 196-219 (2017).

21. Chuaqui, T.R.C., Linear and non-linear vibrations of single-layer graphene sheets, (DEMec, Faculty of Engineering, University of Porto, Porto, 2016).

22. Ribeiro, P., J. Sound Vib. 322(1-2): p. 8-14 (2009).

23. Ribeiro, P. and M. Petyt, Int. J. Non-Linear Mech. 35(2): p. 263-278 (2000).

24. Lewandowski, R., J. Sound Vib. 170(5): p. 577-593 (1994).

25. Sarma, M.S., et al., J. Sound Vib. 159(3): p. 540-545 (1992).

26. Mei, C. and K. Decha-Umphai, AIAA J. 23(7): p. 1104-1110 (1985).

27. Eisley, J.G., J. Appl. Math. and Phys. (ZAMP) 15(2): p. 167-175 (1964). 